A N N A L E S Annales de Bretagne et des Pays de l'Ouest

Anjou. Maine. Poitou-Charente. Touraine

111-3 | 2004

Alcuin de York à Tours

\title{
Autour du De virtutibus et vitiis d'Alcuin
}

\section{Alain Dubreucq}

\section{OpenEdition}

\section{Journals}

Édition électronique

URL : http://journals.openedition.org/abpo/1247

DOI : $10.4000 / a b p o .1247$

ISBN : 978-2-7535-1495-9

ISSN : 2108-6443

Éditeur

Presses universitaires de Rennes

Édition imprimée

Date de publication : 20 septembre 2004

Pagination : 249-288

ISBN : 978-2-7535-0053-2

ISSN : 0399-0826

Référence électronique

Alain Dubreucq, "Autour du De virtutibus et vitiis d'Alcuin », Annales de Bretagne et des Pays de l'Ouest [En ligne], 111-3 | 2004, mis en ligne le 20 septembre 2006, consulté le 02 mai 2019. URL : http:// journals.openedition.org/abpo/1247; DOI : 10.4000/abpo.1247 


\title{
Autour du De virtutibus et vitiis d'Alcuin
}

\author{
Alain DuBREucQ \\ Professeur d'histoire médiévale à l'Université Jean-Moulin (Lyon 3) \\ Directeur du CERCOR (Saint-Étienne)
}

Le Liber de virtutibus et vitiis est un traité d'Alcuin qui a largement suscité l'intérêt de l'historiographie moderne, mais ne bénéficie malheureusement pas aujourd'hui d'une édition scientifique fiable. Force est donc pour l'instant de recourir à la transcription par Migne de l'édition Froben réalisée en 1777 sur la base de trois manuscrits de Ratisbonne, qui améliorait grandement les éditions précédentes de Margerin de la Bigne et de Canisius ${ }^{1}$. Heureusement, une nouvelle édition de l'œuvre est en cours par les soins de Paul Szarmach, lequel a publié en 1981 une liste des très nombreux manuscrits - plus de cent quarante -, qui composent sa tradition ${ }^{2}$. Il s'agit donc d'une œuvre très diffusée, qui est en fait en grande partie un florilège. Ses sources ont été étudiées en premier lieu par Henri Rochais, dont les résultats ont été contestés par Luitpold Wallach, qui soulignait l'héritage isidorien du texte ${ }^{3}$. Récemment, celui-ci a fait l'objet d'un des derniers articles du regretté Donald Bullough, qui met l'accent sur le modèle que propose Alcuin des vertus laïques, dans ses lettres comme dans le De virtutibus et vitiis ${ }^{4}$.

La date exacte de rédaction n'est pas connue et ne peut qu'être estimée. Brunhölzl la plaçait en 799-800, mais il semble préférable de suivre les propositions de Donald Bullough, qui a bien montré que la rédaction du De virtutibus et vitiis était postérieure à celle d'une autre œuvre d'Alcuin, la Disputatio de rhetorica et de virtutibus ${ }^{5}$. En effet, le chapitre 35 du De vir-

1. Alcuin, De virtutibus et vitiis..., d'après Frobenius Flacci Albini... opera, Ratisbonne, 1777.

2. P. E. SZARMACH, "A preliminary handlist... », p. 131-140. L'auteur (p. 132-133) recense plus de 140 manuscrits, depuis l'époque de composition de l'œuvre jusqu'à la fin du Moyen Âge et répartis dans toute l'Europe, avec un grand nombre de traductions en langue vernaculaire. La nouvelle édition préparée par cet auteur se fonde sur le ms. Troyes, Bibl. Munic. 1742, $\mathrm{IX}^{\mathrm{e}}$ siècle, provenant de Clairvaux et comportant la dédicace ad Widonem comitem.

3. H. Rochals, « Le Liber de virtutibus et vitiis... ", p. 77-86; L. WALLACH, "Alcuin on virtues and vices...", p. 175-195.

4. D. A. Bullough, "Alcuin and lay virtue..." ", p. 71-92.

5. Alcuin, Disputatio de rhetorica et virtutibus; $\mathrm{cf}$. D. A. Bullough, "Alcuin and lay virtue..." ", p. 82-83, n. 46. 
tutibus et vitiis reprend en les abrégeant fortement des emprunts presque textuels de la Disputatio au De Inventione de Cicéron. Si l'on considère que la Disputatio est postérieure au départ d'Alcuin d'Aix, le De virtutibus et vitiis peut être placé entre 801 et 804.

Ce traité, auquel le Moyen Âge a attribué une grande importance, est souvent considéré comme un miroir des laïcs, ou plus précisément comme un manuel du soldat chrétien ${ }^{6}$. En fait, toute une conception de la vie chrétienne s'y exprime, fondée sur l'union de la philosophie et de la morale chrétienne, mais appliquée au monde laïque.

\section{L'œuvre et ses sources}

\section{Destination et composition du texte}

Le traité était composé dès l'origine de 35 chapitres comme l'indique la tradition manuscrite et non de 36, chiffre donné par Migne et repris par l'historiographie traditionnelle ${ }^{7}$. De fait, une mention dans le catalogue des manuscrits de Saint-Gall du Ix ${ }^{\mathrm{e}}$ siècle montre que dès cette époque, il n'y en avait que 35 : Alchuini ad Vitonem comitem capitula $X X X V^{8}$. Il se divise en quatre parties distinctes par le style et le contenu; une lettre dédicatoire ouvre le traité et une peroratio le termine, qui a été assimilée à tort à un trente-sixième chapitre $^{9}$. Cependant, le paragraphe qui la suit dans le texte publié par Migne a été ajouté par erreur à l'œuvre d'Alcuin et ne figure pas dans les manuscrits. Les 26 premiers chapitres abordent les sujets suivants : la sagesse, la foi, la charité, l'espérance, la lecture de l'Ecriture sainte, la paix, la miséricorde, le pardon, la patience, l'humilité, le remords, la confession, le repentir, le retour à Dieu, la crainte de Dieu, le jeûne, la chasteté, l'interdiction de la fraude, les devoirs des juges, les faux témoignages, l'envie, l'orgueil, la colère, la tromperie et la persévérance dans les bonnes oeuvres. Les c. 2734 donnent le catalogue des huit vices principaux. Le c. 35 commente les quatre vertus cardinales : sagesse, justice, force et tempérance, qui sont les quatre parties de la virtus. Les deux lettres sont conformes au style épistolaire habituel d'Alcuin, la seconde partie du traité est de style homilétique, la section sur les vices et vertus est purement matérielle et descriptive.

La rédaction du traité semble faire suite à une demande de Guy, comte de la marche de Bretagne et Alcuin le présente comme un breviarium, c'està-dire un manuel composé brevi sermone de chapitres courts, destiné à accompagner le comte dans ses voyages et expéditions militaires : " J'ai dicté ceci pour toi, très cher fils, en termes brefs, comme tu me l'as demandé ${ }^{10}$. "

6. C'est le sous-titre de l'article de L. WALLACH, "Alcuin on virtues and vices... ".

7. H. RochaIS, "Le Liber de virtutibus et vitiis... ", p. 78.

8. P. Lehmann (éd.), Mittelalterliche Bibliothekskataloge..., tome 1, p. 79.

9. La lettre de dédicace et la peroratio finale sont éditées par E. DUMMLER, MGH Ep. IV, Ep. 305, p. 464.

10. Alcuin, De virtutibus et vitiis, PL 101, col. 636 : Haec tibi dulcissime fili brevi sermone, sicut petisti, dictavi. 
Cette requête ne doit pas faire illusion, car elle fait partie d'un procédé de style bien connu de l'époque carolingienne, chez Alcuin lui-même mais aussi dans la plupart des miroirs des princes et des laïcs, comme l'a montré Michel Rouche dans le colloque Commitenti des semaines de Spolète ${ }^{11}$. Par ailleurs, l'aspect homilétique de la seconde section du traité consacrée aux vertus n'a pas échappé au rédacteur anonyme de la Vita Alcuini, qui écrit : "Pour le comte Guy [il écrivit] des homélies sur les principaux vices et vertus ${ }^{12}$." Notons que l'auteur étend cet aspect homilétique à l'ensemble du traité, soulignant ainsi l'intervention personnelle d'Alcuin, et non une demande du comte. La rédaction du traité est donc davantage représentative de la conception alcuinienne de la vie idéale des laïcs que du point de vue de ceux-ci. En effet, dans tout le traité, il n'est plus guère fait allusion à la personne ou à la fonction du comte, sauf dans la peroratio finale. L'ensemble est très général et peut donc être applicable à n'importe quel chrétien. Le contenu en est simple : la vie est le théâtre d'un conflit entre les vertus et les huit vices qui sont à l'origine de tous les maux. Dans ce combat quotidien que doit mener le miles christianus, les vertus sont conduites par quatre duces gloriosissimi, c'est-à-dire les vertus cardinales, qui écrasent les vices, nommés ici les duces impietatis $^{13}$.

Cette métaphore militaire était tout à fait appropriée à cet aristocrate carolingien engagé activement dans la vie militaire auquel Alcuin, à la fin de sa vie, adressa le De virtutibus et vitiis. Dans ce manuel, il associe les deux genres de la lettre d'admonition et du traité de morale politique. C'est ce qu'avait fait avant lui Paulin d'Aquilée, qui adressa vers 795-796 un Liber exhortationis en 66 chapitres au comte et duc Éric de Frioul, autre représentant du pouvoir carolingien dans des terres nouvellement conquises ${ }^{14}$. La similitude de situation d'Éric de Frioul et de Guy de Bretagne est frappante et il faut signaler que le premier faisait aussi partie des correspondants d'Alcuin. Celui-ci lui adressa vers 796 une lettre dans laquelle il lui dit qu'il lui aurait volontiers écrit davantage "sur l'observance de la piété chrétienne s'il n'avait à sa disposition un docteur éminent et un maître de vie céleste ${ }^{15}$ ». Alcuin avait donc connaissance de l'œuvre de Paulin et c'est au même titre de directeur de vie spirituelle qu'il s'adressait à Guy. Cependant, la tonalité de l'exhortation est différente : alors que Paulin insistait sur la vie spirituelle du miles terrenus et appelait à la perfection, condi-

11. M. Rouche, " Miroirs des princes ou miroir du clergé? ", p. 344, 351-352.

12. Vita Alcuini, c. 21, MGH SS XV, p. 195, traduction française par Philippe Depreux et Bruno Judic, communiquée au cours du colloque de Tours en mars 2004 (p. 16).

13. Alcuin, De virtutibus et vitiis, c. 34, PL 101, col. 636 D.

14. Paulin d'Aquilée, Liber exhortationis, dans PL 99, col. 197-282. Sur ce texte, voir le travail récent d'Y. M. DuvAL, "Paulin d'Aquilée et le duc Eric ". La coloration spirituelle et ascétique de cette exhortation est bien marquée par le type monastique des sources utilisées par Paulin : le De vita contemplativa de Julien Pomère, PL 59, col. 415-520 et l'Admonitio Basilii... Noter que Paulin s'adresse à Éric en l'appelant frater, col. 197 C, soulignant ainsi l'égalité du laïc et du clerc au sein du peuple de Dieu.

15. Alc. Ep. 98, p. 142. 
tion de la vie éternelle, Alcuin s'adresse davantage à Guy en tant que comte, titulaire d'un honor et d'une dignité qui lui donnent des prérogatives judiciaires, dans l'exercice desquelles il doit respecter les règles de la morale chrétienne.

La partie homilétique n'est pas à proprement parler un traité des vertus, auxquelles un autre chapitre est consacré à la fin de l'ouvrage, mais plutôt un exposé des exercices spirituels que doit pratiquer le soldat chrétien, à l'exemple des moines, dans la lutte contre les vices qui l'assaillent. Les chapitres XI à XIII, intitulés De compunctione cordis, De confessione et De poenitentia entrent en effet dans cette catégorie ${ }^{16}$.

À la fin de son ouvrage, Alcuin donne des informations sur la forme de celui-ci et sur son usage, qui ne sont pas sans intérêt pour l'étude du genre littéraire ${ }^{17}$. Il s'agit d'un liber manualis ${ }^{18}$, un petit ouvrage transportable et bref, comportant des sententiae réunies en florilège, mais un manuel spécial, car en le lisant on peut se regarder soi-même et donc se connaître. Cette idée chère à la philosophie grecque renvoie dans ce contexte à la métaphore du miroir, même si ce terme n'apparaît pas pour désigner l'écrit d'Alcuin ${ }^{19}$. Elle invite à la connaissance de soi, mais en même temps au désir de la béatitude éternelle. Cette tension entre les deux caractéristiques du miroir est encore précisée dans la peroratio finale, qui évoque le but ultime de l'exercice proposé, atteindre le "sommet de la perfection ». Il est cependant difficile de l'atteindre seul, c'est pourquoi l'élève, ici le comte Guy, a besoin d'un maître spirituel, et c'est tout le sens des relations entre

16. C'est Donald Bullough, "Alcuin and lay virtue..." " p. 85, qui qualifie cette section du De virtutibus et vitiis d'exercices spirituels, de manière tout à fait heureuse à mon avis, car elle rend bien l'aspect monastique du modèle ainsi proposé aux grands laïcs.

17. Lettre de dédicace, PL 101, col. 613 C : [...] ut habeas jugiter inter manus manuales paternae admonitionis sententias, in quibus teipsum considerare debuisses, atque ad aeternae beatitudinis excitare studium; dans la peroratio finale, ibid., col. 638: Haec tibi, dulcissime fili, brevi sermone, sicut petisti, dictavi, ut habeas ea quotidie quasi manualem in conspectu tuo libellum, et in quo possis teipsum considerare, quid cavere, vel quid agere debeas, atque per singulas vitae hujus prosperitates vel adversitates exhortari, quomodo ad culmen perfectionis ascendere debeas.

18. On pense bien sûr au plus connu des miroirs des laïcs, le Liber manualis écrit par la noble Dhuoda. Donald Bullough a suggéré il y a quelques années que le plus ancien témoin du De virtutibus et vitiis, le manuscrit de Troyes, dont les dimensions réduites concordent avec l'idée d'un manuel, pouvait être l'exemplaire destiné au comte Guy, mais il expose lui-même les raisons qui rendent cette attribution douteuse, cf. D. A. BuLLough, "Alcuin's cultural influence... ", p. 20-21.

19. Le De virtutibus et vitiis ne peut être considéré véritablement comme un miroir des laïcs car, bien qu'adressé à l'un d'entre eux, il ne cherche pas à théoriser la condition de celui-ci, ses rapports au monde et avec les autres pouvoirs, ni la condition des laïcs en général. Cette étape ne fut franchie que sous le règne de Louis le Pieux, avec le De institutione laicali de Jonas d'Orléans. La métaphore du miroir n'est ici qu'esquissée et n'est pas poussée dans ses conséquences spécifiques, justifiant le contrôle des laïcs par l'épiscopat. Noter que dans le De virtutibus et vitiis, la fonction de miroir est réservée aux saintes Écritures, en tant que voie d'accès au ciel, De virtutibus et vitiis, c. V, PL 101, col. 616 D : Sanctarum lectio Scripturarum divinae est cognitio beatitudinis. In his enim quasi in quodam speculo homo seipsum considerare potest, qualis sit, vel quo tendat. 
le comte et Alcuin, qui adresse à son " très cher fils " ou "fils très doux " une admonition paternelle (admonitio paterna). Le maître de Tours est un père spirituel, sur le modèle monastique, qui a le devoir d'enseigner, donc d'exhorter son fils spirituel à l'exercice des vertus chrétiennes ${ }^{20}$. On ne s'étonnera pas, dans ces conditions, que l'abbé souligne que son livre « a été dicté par la vigueur d'une sainte charité " : cette caritas, amour fraternel unissant aussi bien les frères au monastère que tous les fidèles au sein de la société des baptisés, se retrouve fréquemment comme motif d'envoi des lettres d'Alcuin aux grands de son époque. L'abbé de Saint-Martin proposait ainsi à ceux-ci un modèle proprement monastique

\section{Les sources du De virtutibus et vitiis}

L'identification des sources du De virtutibus et vitiis a fait l'objet d'un débat historiographique, qui a permis de donner un éclairage neuf sur les méthodes de composition des florilèges carolingiens. Dom Rochais voyait dans le De virtutibus et vitiis une influence prépondérante du Livre des étincelles de Defensor de Ligugé, qui aurait constitué la toile de fond de tout le texte, le reste étant inspiré de sermons pseudo-augustiniens ${ }^{21}$. Cependant, l'étude ultérieure de Luitpold Wallach a montré définitivement que la source essentielle n'était pas l'œuvre de Defensor, mais les Sententiae d'Isidore de Séville, utilisées directement par Alcuin ${ }^{22}$. Par ailleurs, une partie des citations du De virtutibus et vitiis attribuées à des sermons pseudoaugustiniens pourraient bien être l'œuvre d'Alcuin lui-même. Il n'entre pas dans le cadre de cet article d'entreprendre une description systématique des sources du De virtutibus et vitiis, que l'on trouvera dans la prochaine édition du texte, et on se contentera d'en donner brièvement les grandes lignes, pour permettre de comprendre l'esprit de sa rédaction ${ }^{23}$.

La lettre de dédicace est conforme au style épistolaire d'Alcuin et il ne faut pas y chercher de références littérales émanant d'autres sources. La seconde partie, qui commente les attitudes et exercices nécessaires à la vie chrétienne est fortement inspirée des Sentences d'Isidore, dans les chapitres $1-4,11$ et 22 , alors que le chapitre 14 est presque entièrement démarqué du sermon 18, 2-6 de Césaire d'Arles sur la nécessité de se tourner vers Dieu le plus vite possible. Les chapitres 5 à 10 et 12-13 combinent des références à des homélies apocryphes pseudo-augustiniennes, alors que les chapitres 20 et 21, qui concernent essentiellement le noble laïc dans ses

20. Noter que Paulin emploie le terme de frater, et non celui de filius, dans son exhortation, ce qui donne un ton très différent aux relations unissant le clerc au laïc.

21. H. RochaIS, "Le Liber de virtutibus et vitiis... ", p. 77-86.

22. L. WALLACH, "Alcuin on virtues and vices... ", p. 175-195.

23. En attendant la nouvelle édition de P. Szarmach, qui fera le point sur les sources du texte, il faut renvoyer à l'article de L. WALLACH, ibid., auquel le développement qui suit doit l'essentiel, et aux compléments apportés par D. A. Bullough, "Alcuin and lay virtue... ", p. 86-87, notamment en ce qui concerne l'attribution de certains sermons pseudoaugustiniens. 
activités judiciaires est un arrangement de divers extraits des Sentences d'Isidore, livre III, 52-53. Enfin, le chapitre 23, consacré à l'orgueil doit beaucoup au De Vita contemplativa de Julien Pomère.

La troisième partie, qui traite des vices à combattre, se compose de huit chapitres consacrés à chacun des huit vices capitaux. Les sources essentielles sont ici les Moralia de saint Grégoire le Grand, XXXI, 45 et les Conférences de Jean Cassien V, 1 et V, 16.5. Alcuin suit la classification de Jean Cassien, mais Grégoire lui fournit la matière du développement. Le chapitre 35, qui définit la virtus et ses composantes, est évidemment inspiré du De inventione de Cicéron et de l'arrangement qu'avait fait précédemment Alcuin de citations de celui-ci dans son De rhetorica et virtutibus. Il faut noter que, dans l'ensemble du traité, si le ton est souvent augustinien, on ne trouve guère de citations littérales qui puissent être attribuées à l'évêque d'Hippone.

\section{Des vices et des vertus}

\section{Une liste adaptée aux laïcs}

La liste des huit tentations qui menacent l'homme fut formulée pour la première fois par un Père du désert, Evagre le Pontique, diacre puis moine en Égypte dans la deuxième partie du IV ${ }^{\mathrm{e}}$ siècle, dans l'ordre suivant : gourmandise, luxure, avarice, tristesse, colère, acédie, vaine gloire et orgueil ${ }^{24}$. Son élève Jean Cassien incorpora cette liste dans ses propres écrits, en la précisant :

"Il y a huit principaux vices qui font au genre humain la guerre : le premier est la gourmandise ou folie du ventre (gastrimargia); le deuxième la fornication; le troisième l'avarice ou l'amour de l'argent (philargyria); le quatrième la colère; le cinquième la tristesse; le sixième l'acédie ou l'inquiétude et l'ennui du cœur; le septième la vaine gloire (cenodoxia); le huitième l'orgueil $^{25}$."

Entre ces deux formulations, deux différences essentielles se dégageaient déjà : alors que, chez Evagre, il s'agissait de logismoi ou esprits mauvais, qui attaquaient le moine pour l'empêcher d'atteindre l'apatheia et l'éloigner de Dieu, la liste de Cassien se compose de huit vices, que le diable envoie pour tenter l'homme. Par ailleurs l'ordre de la colère et de la tristesse se trouve inversé. Les vices charnels, gourmandise et luxure, mènent fatalement aux vices spirituels, vaine gloire et superbe, un vice plus léger en entraînant d'autres plus graves ${ }^{26}$.

24. Evagre le Pontique, Tractatus..., c. 1145 sqq.

25. J. Cassien, Conlationes V, surtout c. 2, p. 188-190.

26. Sur la constitution des listes médiévales, voir l'excellente synthèse de C. CASAGRANDE et S. Vecchio, Histoire des péchés capitaux..., p. 276 sqq., auquel ce développement doit beaucoup. 
Dans ce cheminement, les vices s'engendrent les uns les autres, en un processus destructeur de l'âme humaine. C'est cependant Grégoire qui constitua définitivement la hiérarchie du système :

" Les vices sont attachés par un lien de parenté très étroit dès lors qu'ils dérivent l'un de l'autre. La première fille de la superbe est en fait la vaine gloire qui, une fois l'esprit vaincu et corrompu, engendre aussitôt l'envie... L'envie engendre la colère... De la colère naît la tristesse... De la tristesse on arrive à l'avarice, puis la gourmandise et la luxure ${ }^{27} \ldots$ "

Dans ce schéma, chacun des vices dérive du précédent et s'exprime à partir de lui. De la sphère érémitique et cénobitique, la liste prenait donc un sens plus général avec Grégoire, qui voyait en l'orgueil la racine de tous les autres vices et distinguait entre les péchés charnels et spirituels. Cette distinction était peut-être perdue dans les Sentences d'Isidore, bien que celui-ci décrivît la superbia comme la reine des vices (II, 37). La liste grégorienne avait l'avantage d'être utilisable pour la pastorale.

En revanche, c'est bien la liste de Cassien qui fournit la trame d'un des principaux pénitentiels insulaires, celui de Cumméan. En effet, celui-ci innove en utilisant la liste de Cassien comme support théorique de sa discussion des fautes commises et de leur punition : les fautes sont l'application pratique des huit vices, qui rendent l'homme malade, et à chaque faute correspond une pénitence, destinée à soigner cette blessure ${ }^{28}$. Ainsi, le cas du laïc qui souille la femme de son prochain, passible d'un an de pénitence, est traité dans le cadre du titre II, De fornicatione, et celui du voleur dans le titre III, De filargiria ${ }^{29}$. Rien ne prouve que ce modèle ait été connu d'Alcuin, mais il est remarquable que celui-ci suit la liste de Cassien, sauf en un cas, et que l'évolution pénitentielle permettait l'application pratique du schéma aux fautes des laïcs. La liste des vices et de leurs conséquences, formulée à l'origine dans un contexte de retrait du monde, visait maintenant les cas auxquels les aristocrates étaient le plus confrontés dans leur vie publique.

Après Grégoire, on assiste à une dilution relative de la distinction entre vices charnels et spirituels, les vices ayant leur origine à l'intérieur même de l'homme, en une filiation certes hiérarchique, mais où ils sont tous au service du péché majeur, l'orgueil. On est donc passé d'un octénaire à un septénaire, l'orgueil occupant une place à part, en tant que racine de tous les maux. Ce système avait été pensé à l'origine par des moines et pour des moines, pour les guider sur le chemin de la perfection. Mais l'époque carolingienne le fit sortir du milieu monastique, avec Alcuin et son De virtutibus et vitiis, pour l'adapter au mode de vie des laïcs et prendre en charge leur comportement.

27. Grégoire le Grand, Moralia, XXXI, 45, 89, p. 1611.

28. Cf. le titre du prologue du pénitentiel : Prologus de medicinae salutaris animarum.

29. Éd. L. BIELER, p. 114-116. 


\begin{tabular}{|c|c|c|c|c|c|c|}
\hline CASSIEN & GREGOIRE & $\begin{array}{l}\text { ISIDORE } \\
\text { Différences }\end{array}$ & $\begin{array}{l}\text { ISIDORE, } \\
\text { Quaest. } \\
\text { in vetus test }\end{array}$ & Alcuin & JONAS & RABAN MAUR \\
\hline $\begin{array}{l}\text { Gourmandise } \\
\text { Luxure } \\
\text { Avarice } \\
\text { Colère } \\
\text { Tristesse } \\
\text { Acédie } \\
\text { Vaine gloire } \\
\text { Orgueil }\end{array}$ & $\begin{array}{l}\text { Orgueil (source) } \\
\text { Vaine gloire } \\
\text { Envie } \\
\text { Colère } \\
\text { Tristesse } \\
\text { Avarice } \\
\text { Gourmandise } \\
\text { Luxure }\end{array}$ & $\begin{array}{l}\text { Gourmandise } \\
\text { Luxure } \\
\text { Avarice } \\
\text { Envie } \\
\text { Tristesse } \\
\text { Colère } \\
\text { Vaine gloire } \\
\text { Orgueil }\end{array}$ & $\begin{array}{l}\text { Gourmandise } \\
\text { Luxure } \\
\text { Avarice } \\
\text { Acédie } \\
\text { Tristesse } \\
\text { Colère } \\
\text { Vaine gloire } \\
\text { Orgueil }\end{array}$ & $\begin{array}{l}\text { Orgueil } \\
\text { Gourmandise } \\
\text { Luxure } \\
\text { Avarice } \\
\text { Colère } \\
\text { Tristesse } \\
\text { Acédie } \\
\text { Vaine gloire }\end{array}$ & $\begin{array}{l}\text { Orgueil } \\
\text { Gourmandise } \\
\text { Luxure } \\
\text { Avarice } \\
\text { Colère } \\
\text { Acédie } \\
\text { Tristesse } \\
\text { Vaine gloire }\end{array}$ & $\begin{array}{l}\text { Orgueil } \\
\text { Vaine gloire } \\
\text { Envie } \\
\text { Colère } \\
\text { Tristesse } \\
\text { Avarice } \\
\text { Gourmandise } \\
\text { Luxure }\end{array}$ \\
\hline
\end{tabular}

La composition de la troisième partie du De virtutibus et vitiis ne semble pas homogène au premier abord : en effet, la superbia est abordée deux fois, aux chapitres 23 et 27, la colère aux chapitres 24 et 31 et les vertus étudiées au début de l'ouvrage sont récapitulées au chapitre 35. Ce désordre n'est qu'apparent et semble répondre à une volonté de concilier la liste monastique de Cassien avec la pensée grégorienne, qui renvoie le débat à l'intérieur de l'homme. Ainsi, le chapitre 24 De iracundia stigmatise une tendance à la colère, qui naît à l'intérieur de l'homme, mais n'est pas forcément mauvaise si elle s'exprime contre ses propres péchés, alors que l'ira du chapitre 31 est un vice qui envahit l'homme, le prive de son jugement et entraîne des conséquences néfastes sur sa vie : querelle, rixe, homicide. Alcuin y ajoute le désir de vengeance et la mémoire des offenses, typiques de la société franque ${ }^{30}$. On est donc passé ici du niveau spirituel au plan très pratique de l'application aux potentes de la cour carolingienne.

Le chapitre 27 est une introduction aux huit vices principaux et primo de superbia. Celle-ci est présentée dans le chapitre 23 comme le plus grand péché du diable, qui naît souvent d'œuvres qui seraient bonnes si l'homme ne s'en glorifiait pas, perdant ainsi par orgueil ce que la charité lui avait fait acquérir. Dans le chapitre 27, la superbia est la reine de tous les maux, à cause de laquelle les anges tombèrent du ciel, quand ils méprisèrent les commandements de Dieu, ce qui provient des Moralia de Grégoire ${ }^{31}$, mais sa définition est tout autre : "La superbe survient par une disposition rebelle, quand les hommes négligent d'obéir à leurs seniores ${ }^{32}$. " Là encore, Alcuin passe du domaine spirituel à celui du système social carolingien et au problème de la rébellion éventuelle des grands laïcs. Alcuin pensait peutêtre même à un cas précis puisque les rébellions n'avaient pas manqué à la fin du VIII ${ }^{\mathrm{e}}$ siècle, de Tassilon à Pépin le Bossu ${ }^{33}$. On peut étendre ce type d'analyse aux autres chapitres mentionnés plus haut mais, faute de place, il n'est pas possible de le faire ici.

30. De virtutibus et vitiis, c. 31 , col. 634 .

31. PL 101, c. 633 A, cf. Moralia XXXI, 45, 87, p. 1610 (cf. Isidore, Sentences. 37, 8, p. 167 et 38,2 , p. 168).

32. De virtutibus et vitiis, c. 27, col. 633 B : Fit etiam per contumaciam, quando despiciunt homines senioribus oboedire suis.

33. On peut voir un écho de ce problème dans le Capitulare missorum Aquisgranense primum de 810, c. 17, MGH Capit. I, $\mathrm{n}^{\circ} 64$, $\$ 153$ : Ut unusquisque suos juniores distringat, ut melius et melius oboediant et consentiant mandatis et praeceptis imperialibus. 
La liste des péchés capitaux, on l'a vu, est reprise de Cassien, mais avec l'orgueil en tête. La liste de Grégoire faisait pourtant autorité aux VII ${ }^{\mathrm{e}}$ $\mathrm{VIII}^{\mathrm{e}}$ siècles. Il est donc intéressant de comprendre pourquoi et comment Alcuin renoue avec la tradition ancienne. En fait, le De virtutibus et vitiis marque l'instant d'une fusion entre la pensée purement monastique de Cassien et celle, plus ouverte sur le monde, de Grégoire. C'est pourquoi il tient compte de la prééminence donnée par celui-ci à l'orgueil en le plaçant en tête de liste. D'autre part, la liste de Grégoire mentionne l'envie en troisième position des vices, alors que Cassien ne voyait dans l'envie que l'un des rejetons de l'orgueil. Devant cette situation, Alcuin propose un compromis : il consacre un chapitre entier à l'envie, comme Grégoire, mais il ne l'introduit pas dans la liste de Cassien, préférant le placer avant l'étude des huit vices principaux ${ }^{34}$. Ce faisant, il respectait l'aspect monastique de la liste ancienne, tout en prenant en compte la réflexion grégorienne, et faisait de l'étude de l'envie une sorte de préambule à celle des autres vices. Par ailleurs, le traitement par Alcuin des péchés secondaires issus des huit vices principaux dérive pour l'essentiel de Grégoire. Il faut noter cependant que cette tentative de fusion des modèles resta sans lendemain, puisque dès la génération suivante, avec Jonas d'Orléans, on revint au respect strict de la liste grégorienne ${ }^{35}$.

\section{L'acédie, un vice lä̈c?}

Il est singulier que l'acédie trouve place dans un traité adressé à un laïc du début du IX ${ }^{\mathrm{e}}$ siècle. Il s'agissait au départ d'un vice proprement lié à la vie érémitique, né dans le cadre du monachisme érémitique oriental du IV siècle et défini par Evagre le Pontique ${ }^{36}$ comme la gêne et l'angoisse du cœur aboutissant à une faiblesse de l'âme. Avec Jean Cassien, ce vice se cénobitisa et, ce faisant, connut une évolution majeure : le lien avec la tristesse, qui éloigne l'homme de la voie de la contemplation. L'acédie, engendrée par la tristesse, engendre elle-même d'autres vices : oisiveté, somnolence, mauvaise humeur, inquiétude, vagabondage ${ }^{37}$. Le travail manuel en est le seul remède. Cassien, accorde donc une place majeure aux conséquences du vice et à son affinité avec la tristesse. Un siècle plus tard, avec Grégoire, l'acédie tend à disparaître pour n'être plus qu'un rejeton de la tristesse. Ceci semble correspondre, selon Carla Casagrande et Silvana Vecchio, à un nouveau type de monachisme, qui se coupait de la tradition orientale : on vit en communauté, sous une règle, en alternant travail, étude et prière, mode de vie qui marginalise l'acédie ${ }^{38}$.

34. De virtutibus et vitiis, c. 22, col. 630 B.

35. Sur cette évolution, consulter R. WASSELYNCK, "Les Moralia in Job... ", p. 19.

36. Evagre le Pontique, Tractatus..., col. 1158-1159.

37. Jean Cassien, Institutions, p. 382-425. Voir le chapitre consacré à l'acédie par C. CASAGRAnde et S. Vecchio, Histoire des péchés capitaux..., p. 128-144.

38. Ibid., p. 130-131. 
Celle-ci va réapparaître dans le De virtutibus et vitiis d'Alcuin, mais avec une inflexion capitale : elle va quitter le monde monastique pour s'appliquer aux laïcs. Sa nature est cependant différente de l'acédie monastique, car l'inquiétude spirituelle s'y estompe au profit des aspects physiques. Alcuin en souligne ainsi les conséquences :

"La somnolence, la fainéantise dans les bonnes oeuvres, l'incapacité à tenir en place, le vagabondage d'un endroit à un autre, l'indolence au travail, la lassitude du cœur, les lamentations et les discours inutiles ${ }^{39}$."

Elle se rapproche donc de l'oisiveté, otiositas, détournement du sens premier du concept romain d'otium, qui caractérisait le mode de vie aristocratique et permettait la réflexion philosophique, sans connotation péjorative.

C'est ainsi qu'Alcuin fait le lien entre l'acédie et le monde laïc :

"L'acédie est une peste, qui nuit beaucoup aux serviteurs de Dieu, quand l'homme oisif (otiosus) s'engourdit dans les désirs de la chair. "

Alcuin admet certes un peu plus loin que ce mal frappe particulièrement les moines et les chasse de leur cellule, mais tout homme oisif y est aussi exposé. Selon Carla Casagrande et Silvana Vecchio, c'est Jonas d'Orléans qui va faire le lien définitif dans le De Institutione laicali ${ }^{40}$, suivi de Raban Maur ${ }^{41}$. Il me semble cependant que le chapitre 32 du De virtutibus et vitiis établit déjà ce lien qui, du reste, avait peu avant lui été formulé dans le pénitentiel de Cumméan, qui unit l'acédie, l'oisiveté et la somnolence ${ }^{42}$. Le chapitre du De virtutibus et vitiis sur l'acédie s'insère dans un ensemble, à côté de ceux qui sont consacrés à l'orgueil, au caractère colérique, à la gourmandise et à la luxure, qui vise particulièrement un mode de vie typique de l'aristocratie franque. L'acédie de la morale carolingienne a ainsi perdu beaucoup de son caractère original de vice spirituel engendré par la tristesse pour revêtir l'image d'un péché matériel, qui se traduit par l'oisiveté et la recherche du plaisir, éloignant le laïc de ses responsabilités sociales. Le fait qu'Alcuin lui consacre un chapitre entier du De virtutibus et vitiis, alors qu'il aurait pu, comme Grégoire, l'incorporer dans la tristesse, annonce déjà l'idée de la prise en charge du mode de vie laïc par l’Église carolingienne.

39. De virtutibus et vitiis c. 32, col. 635 A-B. La traduction est celle de C. CASAGRANDE, S. VECCHIO, Histoire des péchés capitaux..., p. 144.

40. Jonas d'Orléans, De Institutione laicali, III, 6, PL 106, col. 245-246 : acedia vel otiositas.

41. Raban Maur, De ecclesiastica disciplina III, PL 112, col. 1251-1253 : « Levé le matin de son lit après une nuit d'ébriété, il ne se consacre à aucune tâche utile, ne va pas prier à l'Église, ne se hâte pas d'aller écouter la parole de Dieu, ne se préoccupe pas de faire l'aumône... de réconforter la victime d'une injustice, préférant aller à la chasse, rester chez lui à provoquer des rixes et des disputes, jouer aux dés, se passionner pour des histoires ou des jeux inutiles, jusqu'à ce que soit prêt son repas, entre-temps préparé par des serviteurs laborieux ", trad. C. CASAGRANDE, S. VECCHIO, Histoire des péchés capitaux, p. 144.

42. Pénitentiel de Cumméan c. 7, p. 120 : De accidia. 1. Otiosus opere extraordinario oneretur et somnolentus vigilia propensiori. 


\section{Le combat des vices et des vertus}

Alcuin, s'adressant à un soldat, lui montre l'armée de l'impiété, commandée par les huit vices principaux, qualifiés de duces, montant à l'assaut du genre humain. À cette armée des vices s'oppose victorieusement celle des vertus saintes des soldats du Christ ${ }^{43}$. Cette image du combat spirituel n'était pas nouvelle, car elle tire son origine d'un traité du poète chrétien Prudence, la Psychomachie, écrite à la fin du IV siècle ${ }^{44}$. Celle-ci mettait en scène l'affrontement des sept vices païens et d'autant de vertus chrétiennes, reprenant l'idée stoïcienne de la morale comme théâtre du combat entre vices et vertus.

Grégoire, dans les Moralia in Job, donna à cette image une coloration proprement militaire, connue de tout le Moyen Âge :

"Parmi les vices qui nous tentent et qui mènent contre nous une guerre invisible sous la conduite de l'orgueil, d'aucuns avancent à la tête de l'armée, en tant que commandants, d'autres suivent tels de simples soldats. En fait, toutes les fautes n'attaquent pas le cœur de la même façon, mais tandis que les plus graves, en nombre limité, vainquent l'esprit en profitant de sa distraction, les innombrables fautes moins graves se répandent. Et à peine la reine même des vices, la superbe, s'est-elle emparée pleinement du cœur après l'avoir vaincu qu'elle le livre aux dévastations des sept vices principaux, qui sont pour ainsi dire ses lieutenants. À la suite de ces commandants arrive l'armée, puisqu'il n'est pas douteux qu'en eux trouvent leurs origines de brutales multitudes de vices ${ }^{45}$."

Par la suite, Isidore de Séville, dans le chapitre II, 33 des Sententiae, démarqua la pensée de Grégoire, mais en complétant le paysage du combat des vices et des vertus par l'opposition d'une vertu particulière à chaque vice $^{46}$. En outre, Aldhelm de Malmesbury, au VIII siècle, mettait à la tête de l'armée du mal les huit vices de la liste de Cassien, qui dirigeaient leurs cohortes respectives ${ }^{47}$. En fin de compte, Alcuin fait la synthèse de ces divers héritages en gardant l'image militaire de Grégoire, le nombre huit de Cassien et l'opposition isidorienne. Ainsi :

"L'orgueil est vaincu par l'humilité, la gourmandise par l'abstinence, la fornication par la chasteté, l'avarice par l'abstinence, la colère par la patience, l'acédie par l'assiduité aux bonnes œuvres, la mauvaise tristesse par la joie spirituelle et la vaine gloire par l'amour de Dieu ${ }^{48}$."

43. De virtutibus et vitiis, c. 34, col. 636 : Hi sunt octo totius impietatis duces cum exercitibus suis et fortissimi contra humanum genus diabolicae fraudis bellatores... Isti vero Deo auxiliante facillime vincuntur a bellatoribus Christi per virtutes sanctas.

44. Prudence, Psychomachie contre Symmaque, éd. Maurice Lavarenne, Paris, Les Belles Lettres (Collection des Universités de France, 62), 1948.

45. Grégoire le Grand, Moralia..., XXXI, 45, 87, p. 1610.

46. Isidore de Séville, Sententiae, II, 33, col. 638 : Contra singula vitia virtutes singulae opponuntur : contra luxuriam, munditia cordis, $[. .$.$] contra odium dilectio [. .$.$] contra ira-$ cundiam patientia.

47. Aldhelm de Malmesbury, De virginitate.

48. De virtutibus et vitiis, c. 34, col. 637. 
Par ailleurs, à la fin de chacun des chapitres consacrés aux vices, Alcuin fait appel aux Sentences d'Isidore pour conclure par le triomphe de la vertu correspondante.

\section{Vertus et attitudes morales}

L'image du vice individuel comme chef militaire à la tête d'une troupe considérable, déployée par Grégoire et complétée par Alcuin, convenait particulièrement à un manuel destiné à un comte servant sur la frontière. Elle sert de transition pour aborder le chapitre final dédié aux quatre vertus, les " quatre chefs (duces) très glorieux " opposés aux " combattants de l'impiété du diable ${ }^{49}$ ". Ces ducs sont les quatre vertus cardinales, prudence, justice, courage, tempérance, dont l'étude forme le chapitre 35, couronnant ainsi l'œuvre d'Alcuin ${ }^{50}$. Ce modèle de la philosophie antique ressurgissait après plusieurs siècles de discrétion et, comme l'a montré Sibylle Mähl, c'est Alcuin qui est allé le plus loin à son époque dans la formulation d'une philosophia moralis, en intégrant les quatre vertus cardinales dans le discours chrétien ${ }^{51}$.

Dans le De virtutibus et vitiis, il s'agit d'une version abrégée de leur description dans le De Rhetorica et virtutibus, avec néanmoins quelques changements notables. Comme dans le De Rhetorica, où il répondait à la demande de Charlemagne d'une définition de la virtus elle même, Alcuin s'inspire du De inventione de Cicéron, mais il en modifie les termes pour mettre en valeur le sens de la virtus dans la vie chrétienne : Virtus est animi habitus, naturae decus, vitae ratio, morum pietas, cultus divinitatis, honor hominis, aeternae beatitudinis meritum ${ }^{52}$, alors que le texte de Cicéron disait seulement : virtus est animi habitus, naturae modo atque rationi consentaneus ${ }^{53}$, ce qu'Alcuin interprétait ainsi dans le De Rhetorica : Virtus est animi habitus, naturae decus, vitae ratio, morum nobilitas. On voit donc qu'Alcuin n'a gardé réellement de Cicéron que la notion d'habitus, en ajoutant les qualificatifs moraux chrétiens appropriés. En outre, dans le De virtutibus et vitiis, il a préféré l'expression de morum pietas à celle de morum nobilitas qui figurait dans sa réponse à Charlemagne. C'est qu'en fait il a transféré cette notion de noblesse dans sa définition de la justice, un peu plus loin ${ }^{54}$. Ce déplacement introduisait la noblesse d'âme dans le domaine de la justice, fonction centrale de l'honor du noble carolingien. Cet exemple illustre la méthode de travail d'Alcuin, faite de retouches successives effectuées en fonction des différents destinataires de ses textes.

49. De virtutibus et vitiis, c. 34 , col. $637 \mathrm{~A}$.

50. De virtutibus et vitiis, c. 35, col. 637 B-D.

51. S. MAHL, Quadriga virtutum..., p. 89-125.

52. De virtutibus et vitiis, c. 35 , col. 637 B : « La vertu, c'est la disposition de l'âme (habitus), la dignité du caractère humain, la raison de vivre, la noblesse des mœurs, le culte de Dieu, l'honneur de l'homme et le mérite du bonheur éternel ".

53. M. Tullius Cicero, De inventione, II, 53-54, éd. F. Stroebel, Leipzig, 1915, p. 145-149.

54. De virtutibus et vitiis, c. 35 , col. 637 C : Justitia est animi nobilitas, alors que la définition de la justice dans le De Rhetorica porte : Justitia est habitus animi. 
Par ailleurs, le développement des différentes parties composant la justitia, qu'Alcuin avait repris de Cicéron dans le De Rhetorica, ne figure plus dans le De virtutibus et vitiis ${ }^{55}$, mais on a vu plus haut qu'un des attributs de la justice, le cultus divinitatis se trouvait ajouté dans celui-ci à la définition de la virtus. Alcuin avait fait suivre la définition cicéronienne de la justice des mots suivants : in hac (justitia) divinitatis cultus et humanitatis iura et aequitas totius vitae conservatur, qui ne figuraient pas chez Cicéron, mais donnaient une valeur proprement chrétienne à cette vertu. Dans le De virtutibus et vitiis, il garde cette valeur, mais intercale entre humanitatis iura et aequitas totius vitae un nouvel élément : et justa judicia ${ }^{56}$. Ce dernier ajout était spécialement adapté à la fonction judiciaire du comte, traitée auparavant dans le chapitre 20 De Judicibus. De même, la définition de la fortitudo dans le De virtutibus et vitiis dépasse celle du De Rhetorica, car elle introduit les vertus de patience et de persévérance et surtout la victoire contre les vices, qui fait écho à la fin du chapitre précédent du De virtutibus et vitiis, lequel présentait la bataille des vices et des vertus ${ }^{57}$. La victoire, but de tout guerrier, est ici associée au courage dans le combat quotidien du laïc chrétien. Par ailleurs, dans le De Rhetorica, Alcuin avait démarqué la définition cicéronienne de la temperantia mais, dans le De virtutibus et vitiis, il change de registre en soulignant le caractère noble de cette vertu et son lien avec l'honor humain, adaptant ainsi son discours à un public aristocratique ${ }^{58}$.

La présentation des vertus cardinales antiques, réinterprétées et christianisées, couronnait en quelque sorte le contenu du De virtutibus et vitiis. Cependant, Alcuin avait commencé celui-ci par un chapitre préliminaire, repris plus tard dans une homélie de Raban Maur ${ }^{59}$, sur la sagesse, la vera sapientia, qui éloigne les hommes du service du diable et les ramène vers Dieu par l'observation des commandements. On ne trouvera dans ce très court chapitre nul développement philosophique ou théologique, tel celui que fit Smaragde un peu plus tard ${ }^{60}$, mais un simple précepte : ne pas faire le mal, mais au contraire faire le bien. Les trois vertus théologales de foi,

55. Ces transformations ont été remarquées par D. Bullough, Alcuin and lay virtue..., p. 89; nous y apportons ici quelques compléments, pour mettre en évidence la nouvelle orientation qu'Alcuin donne dans le De virtutibus et vitiis.

56. De Rhetorica, c. 44 : In hac divinitatis cultus et humanitatis iura et aequitas totius vitae conservatur; De virtutibus et vitiis, c. 35 : In hac divinitatis cultus et humanitatis iura et justa judicia et aequitas totius vitae conservatur.

57. De Rhetorica, c. 45 : Fortitudo est magno animo periculorum et laborum perpessio; De virtutibus et vitiis, c. 35 : Fortitudo est magna animi patientia et longanimitas et victoria contra omnium vitiorum genera.

58. De virtutibus et vitiis, c. 35 , col. $637 \mathrm{D}$ : Nobilis virtus est valde temperantia, per quam omnis honor vitae hujus inter homines constat. Cf. aussi Alc. Ep. 209, p. 347, in omni re temperentia servanda est, quae est via regia totius vitae nostrae.

59. De virtutibus et vitiis, c. 1, col. 614 D-615 A; Raban Maur, Homélie 48, PL 110, col. 8890, cf. la contribution de Michel Perrin dans ce même volume.

60. Smaragde de Saint-Mihiel, Via regia, c. 4, PL 102, 940 s. Ce chapitre est le plus long du texte. 
charité et espérance, qui prennent la suite, ne sont d'ailleurs pas davantage développées. Cependant, en conclusion du chapitre sur la sagesse, le ton se fait plus spirituel : la sagesse en tant que cognitio divinitatis virtus boni operis est, virtus boni operis fructus est aeternae beatitudinis ${ }^{61}$. On ne s'étonnera pas de retrouver la dernière partie de cette formulation dans le chapitre 35, insérée dans la définition d'origine cicéronienne de la virtus sous la forme aeternae beatitudinis meritum ${ }^{62}$. D'ailleurs, Alcuin revient dans ce même chapitre, après avoir donné une définition chrétienne des vertus cardinales, sur l'idée de la sagesse comme intelligence des choses divines : Nulla melior est sapientia, quam ea qua Deus secundum modulum humanae mentis intelligitur et timetur, et futurum ejus creditur judicium. Ainsi, la sagesse trouvait place au début et à la fin du discours des vices et des vertus.

\section{La justice et la pénitence}

\section{Justice et équité}

Dans le De virtutibus et vitiis, Alcuin cherche à susciter chez les potentes laïcs un sens de leur responsabilité morale adapté à leur situation de commandement ${ }^{63}$. Cette responsabilité s'exprime avant tout dans la justice, sur laquelle Alcuin insiste bien plus que sur les vertus spirituelles ou sur l'importance de la prière. Ce thème essentiel de tous les miroirs des princes postérieurs est cependant traité par Alcuin sur un plan purement pratique, dans deux chapitres intitulé De judicibus, et non De Justitia, et De falsis testibus. C'était se placer d'emblée davantage au niveau de l'exercice de la fonction qu'à celui de la mission. Dans le premier, Alcuin insiste sur l'équité du jugement, qui doit être rendu sans acception de personne et sans demander de cadeaux (munera) pour faire justice. Cette idée traditionnelle s'appliquait tout naturellement à un fléau de la société carolingienne, la corruption, à laquelle s'opposaient déjà les premiers capitulaires de Charlemagne et que dénonça Théodulfe d'Orléans dans sa Paraenesis ad judices ${ }^{64}$. Alcuin souligne le dommage que cette corruption infligeait aux pauperes, comme le fit aussi Smaragde au début du règne de Louis le Pieux ${ }^{65}$.

Le chapitre suivant, sur le faux témoignage, visait tout autant les abus de la société carolingienne : Alcuin y soulignait ainsi que le faux témoignage était issu le plus souvent de la peur d'une potestas supérieure ou répondait à la volonté des puissants de protéger leurs amis ou leur parenté contre les rigueurs de la justice, mais que, de toute manière, les pauperes en demeuraient toujours les victimes ${ }^{66}$. Même s'il faut faire la part des larges emprunts que fait Alcuin aux Sentences d'Isidore, son intention était claire :

61. De virtutibus et vitiis, c. 1 , col. $615 \mathrm{~A}$.

62. Ibid., c. 637 B.

63. L. WALLACH, "Alcuin on virtue and vices... ", p. 235.

64. Théodulf, Paraenesis ad judices.

65. Smaragde de Saint-Mihiel, Via regia, c. 28, PL 102, c. 966.

66. De virtutibus et vitiis, c. 21 , col. 629 CD-630 A. 
faire passer au comte, dans l'exercice de sa fonction de juge, le minimum exigé par l'éthique chrétienne, l'équité du jugement. Il restait cependant très pratique, sachant bien que dans la réalité les grands étaient exposés en permanence à enfreindre les règles de la vie chrétienne. D'où un groupe de chapitres consacrés à la componction du cour, à la confession et à la pénitence.

\section{Pénitence et miséricorde}

On retrouve dans le De virtutibus et vitiis les trois thèmes alcuiniens de la pénitence jugement, de la pénitence remède et de la pénitence conversion $^{67}$. Tout d'abord, s'adressant à un judex, il met en valeur une conception pratique et presque juridique de la pénitence : le Christ est juge du pécheur au jugement dernier, comme le montrent les chapitres 7 et 8 du $D e$ virtutibus et vitiis, qui traitent de la miséricorde et de l'indulgence de Dieu. La sévérité du jugement et la miséricorde sont ici des notions liées, mais la responsabilité de l'homme envers son propre jugement est essentielle : pour mériter la miséricorde, il faut se maîtriser soi-même et être miséricordieux envers les autres. La miséricorde divine devient donc fonction de la miséricorde humaine ${ }^{68}$. Le jugement de Dieu est certes tempéré par la miséricorde, mais celle-ci est conditionnelle, sinon le pécheur pourrait persévérer dans son attitude fautive. Alcuin place ainsi sa morale à un plan très matériel, utilisable dans la pratique quotidienne. La notion de jugement ellemême est liée à celle de la miséricorde divine : le pécheur est jugé en fonction de ses actes et lui même peut influer sur son jugement futur par une conduite appropriée ${ }^{69}$. Le même mouvement d'idées est exprimé dans le chapitre 20, De judicibus, à l'aide d'un extrait des Sentences d'Isidore :

"Qui juge dans la droiture porte une balance en mains; dans l'un des plateaux il porte la justice, dans l'autre la miséricorde; par la justice, il exprime la sentence du péché, qu'il tempère par la miséricorde afin que, en un juste équilibre, il réprime par esprit de justice, mais pardonne par miséricorde ${ }^{70}$."

67. Je reprends ici les termes de Michael S. DRISCOLL, Alcuin et la pénitence carolingienne..., p. 152. Donald Bullough a émis des critiques très sévère envers cet ouvrage, dans "Alcuin and lay virtue... ", p. 81, n. 40, mais celui-ci reste la seule synthèse des idées d'Alcuin sur la pénitence. On peut aussi se référer plus généralement aux pages relatives à cette question dans A. ChelINI, L'aube du Moyen Âge..., p. 395-441.

68. LVV 7, PL 100, c. 618 : In judice misericordia et disciplina debet esse : quia una sine altera bene esse non possit. Nam misericordia sola si fuerit, securitatem facit peccandi subjectis. Iterum, si disciplina sola semper aderit vertitur animus delinquentis in desperationem, et judex non merebitur a Deo misericordiam : sed hanc misericordiam a seipso debet homo incipere; c. 617 : Non potest peccator a Deo misericordiam sperare, qui misericordiam non facit sperantibus in se.

69. De virtutibus et vitiis, c. 8, col. 618 : Igitur ex nostro judicio judicat nos Deus, et quammodo in potestate nostra est, quomodo judicemur a judice Deo. Si misericorditer judicamus de in nos delinquentibus, misericorditer judicabit Deus de nobis in se peccantibus.

70. Isidore de Séville, Sentences, III, 52, 4, col. 724 B. 
Valeurs ecclésiastiques et laïques sont donc ici intimement liées, ce que met en évidence la formule sententia peccati. Il est significatif à ce propos que cet extrait d'Isidore soit repris un peu plus tard par Smaragde de Saint-Mihiel dans la Via regia, dans le même contexte de transposition du rôle de la potestas au plan religieux, le but ultime étant la protection des pauperes $^{71}$.

\section{Pénitence et retour à Dieu}

Alcuin, dans les chapitres 12 (De confessione) et 13 (De poenitentia) du De virtutibus et vitiis, expose un véritable processus de dynamique pénitentielle : le pécheur est un malade qui a besoin de secours spirituel, qu'il trouve après la confession dans le remède de la pénitence ${ }^{72}$. Cette conception avait déjà une longue histoire : dans l'Écriture déjà, le Christ est présenté comme médecin, dans les Évangiles de saint Marc, 2, 17 et de saint Luc, 5,31 , qui considèrent les pécheurs comme des malades à guérir. Les pénitentiels s'étaient fait rapidement l'écho de cette notion ${ }^{73}$.

Cependant, l'aspect essentiel du processus pénitentiel est la conversion à Dieu. Dans le chapitre 13 du De virtutibus et vitiis, Alcuin insiste sur la qualité du sentiment intérieur qui pousse l'homme au repentir, dont la sincérité importe davantage que la durée de la pénitence :

"La vraie pénitence ne se mesure pas au nombre d'années, mais dans l'amertume de l'âme... La pénitence, même de courte durée, si elle remue le fond du cœur d'amertume, n'est pas dédaignée de Dieu, le juge juste, qui examine attentivement le secret du cœur. Dieu demande moins la durée qu'il ne pèse la sincérité du pénitent ${ }^{74}$."

La vraie pénitence, et cela suppose qu'il y en ait de fausses, est donc fonction de la sincérité du pécheur et de l'attitude positive de son cœur. C'est une attitude intérieure qui pousse au regret et à la confession, considérée comme un sacrifice d'expiation des fautes : "Celui qui fait miséricorde offre à Dieu un sacrifice de salut qui lui plaît ${ }^{75}$. " Cette attitude suppose non seulement le repentir mais un changement de conduite pour l'avenir : convertir son cœur et éviter de pécher sont les conditions du pardon, mais il faut se confesser tant que l'on vit, car après la mort il est trop

71. Smaragde de Saint-Mihiel, Via regia, c. 28, col. 966.

72. De virtutibus et vitiis, c. 12 , col. 621 B : Hortatur nos saepius Scriptura ad medicamentum fugere confessionis et, un peu plus loin, col. 622 B, il conclut : Salus aegroti unicum est viribus nostris medicamentum cum poenitentia.

73. Pénitentiel de Cumméan, prologue, p. 108; cf. C. VoGEL, Les « Libri paenitentiales »..., p. 70 .

74. De virtutibus et vitiis, c. 13, col. 622 C. : Poenitentia vera non annorum numero censetur sed amaritudine animi... Poenitentia, quamvis sit exigui temporis, si intima cordis amaritudine agitur, non despicitur apud judicem justum Deum, qui cordis secreta considerat. Non enim longitudinem temporis tantum requirit Deus, quantum affectum sinceritatis poenitentis pensat.

75. De virtutibus et vitiis, c. 7, col. 618 B, à rapprocher de Alc. Ep. 131, p. 195 : exspectat Dominus a nobis confessionis sacrificium. 
tard, quand vient le temps du châtiment ${ }^{76}$. D'où la nécessité de la componction du cour, qui conditionne ce retour à Dieu; cette expression a aussi une longue histoire : issue des Pères orientaux, elle a été définie en Occident par saint Grégoire le Grand. Ici, il s'agit d'une douleur de l'âme, causée par le péché, mais aussi par le désir de retrouver Dieu, dont les fautes ont éloigné le pécheur. C'est le thème du chapitre 11 du De virtutibus et vitiis, dans lequel Alcuin souligne que la componction du cœur procède de la vertu d'humilité. Il met ainsi en valeur une dynamique de la conversion intérieure, un mouvement qui va de l'humilité à la componction du cour, puis à la confession et à la pénitence, l'ensemble répondant à une triple définition : l'humilité du cœur, la conscience des péchés et la peur du jugement ${ }^{77}$. La sincérité de ce mouvement se révèle par la profusion des larmes, qui fait mériter l'indulgence divine :

" De la double source de la componction s'écoulent les larmes, d'une par en scrutant attentivement les mérites de ses œuvres, d'autre part en soupirant du désir de la vie éternelle ${ }^{78}$."

Sous un vice peuvent se dissimuler des conséquences positives. Ainsi la componction du cœur peut avoir un lien avec la tristesse : dans le chapitre 33, Alcuin fait la distinction entre la tristesse salutaire et la tristesse pestifère. La tristesse est salutaire si l'on s'attriste de ses péchés suffisamment pour se confesser, faire pénitence et se tourner vers Dieu. L'autre est la tristesse du siècle, qui perturbe l'âme et la fait désespérer. Elle est vaincue par la joie spirituelle, l'espérance et la consolation des Écritures ${ }^{79}$. Il existe de même une bonne et une mauvaise colère. La seconde est celle qui perturbe l'esprit et empêche la décision juste, mais la première est salutaire, si l'homme s'irrite contre lui-même de ses propres péchés et d'avoir péché ${ }^{80}$. Ces distinctions, caractéristiques du style d'Alcuin, visaient à ne pas imposer au comte un modèle trop pessimiste et décourageant, qui aurait rendu inutile la lecture même de ses exhortations. La morale qu'il proposait était nuancée, pratique et laissait toujours une possibilité de recours, même en cas d'action grave, pourvu que l'on se tournât vers Dieu. Elle était donc particulièrement adaptée aux nobles carolingiens, que les activités militaires et judiciaires exposaient souvent à enfreindre les règles de la vie chrétienne.

Le De virtutibus et vitiis est bien comme le disait Wallach un manuel du soldat chrétien destiné au comte Guy, mais c'est aussi plus largement un

76. De virtutibus et vitiis, c. 12, col. 621-CD.

77. De virtutibus et vitiis, c. 11 , col. 620 : Compunctio cordis ex humilitatis virtute nascitur; de compunctione confessio peccatorum; de confessione penitentia; de penitentia vera proveniet delictorum indulgentia. Compunctio cordis est humilitas mentis, cum lacrimis et recordatione peccatorum et timore judicii.

78. De virtutibus et vitiis, c. 11, col. 619 D : Ex gemino fonte compunctionis solent profluere lacrymae... promissio indulgentiae quam habemus a Deo, lacrymas poenitentiae excitat cordi nostro.

79. De virtutibus et vitiis, c. 33 , col. 635 C.

80. De virtutibus et vitiis, c. 24, col. 631 B. 
guide destiné à tous les laïcs, c'est-à-dire les potentes du royaume carolingien. Le mérite essentiel de l'œuvre est d'avoir contribué à établir une morale des laïcs, prenant en compte les exigences spécifiques de leur condition, en matière militaire et judiciaire, en intégrant celles-ci dans une éthique chrétienne. Il n'y a cependant pas de différence de nature entre la vie que laïcs et ecclésiastiques doivent mener : il faut vivre selon l'Évangile, comme l'annonce Alcuin dès la préface du De virtutibus et vitiis. Alcuin, comme dans certaines de ses lettres, fait ici office de directeur spirituel des laïcs comme des clercs. Dans la dédicace du De virtutibus et vitiis, il propose ainsi une véritable intégration du mode de vie laïc dans la sainteté chrétienne :

"Que ton état de laïc et ton genre de vie profane ne t'effrayent pas, comme si dans cet état, tu ne pouvais franchir les portes de la vie éternelle. De même qu'à tous également le royaume de Dieu a été prêché, de même pour les deux sexes, pour tous les âges et toutes les personnes également, selon la dignité de leurs mérites, la porte du royaume de Dieu s'ouvrira. Là, il n'y a pas de distinction entre celui qui dans notre monde était laïc ou celui qui était clerc, entre le riche et le pauvre, entre le jeune (junior) et le vieux (senior), entre l'esclave et le maitre; mais chacun, selon le mérite de ses bonnes oeuvres, sera couronné de la gloire éternelle ${ }^{81}$."

Dans le traité, certains chapitres sont à l'évidence destinés à élaborer une morale spécifique aux laïcs, tels ceux sur la justice ou le faux témoignage, mais on peut considérer que les chapitres traitant de l'orgueil, de la colère ou de l'envie, ou l'inutilité de la vengeance, visent aussi le mode de vie des grands. Au niveau du contenu, c'est une spiritualité de type monastique qui est proposée à ceux-ci. L'introduction de l'acédie dans les vices qu'ils doivent éviter en est l'illustration. L'idée que la même règle s'impose à tous est par ailleurs tout à fait significative de la normalisation carolingienne : elle se retrouve dans les textes narratifs, la liturgie, mais aussi le droit civil et le droit canonique. C'est une idée qui influença un peu plus tard d'autres clercs carolingiens, tel Jonas d'Orléans, mais celui-ci, s'il acceptait l'idée que tous les ordres de la société devaient vivre selon une règle, précisait que chaque ordre devait avoir sa propre règle ${ }^{82}$.

Par ailleurs, Alcuin proposait aux grands une attitude spirituelle, faite de conversion permanente à Dieu, et le combat des vices et des vertus, dont l'âme humaine est le théâtre, était l'occasion pour lui d'insister sur l'attitude personnelle du chrétien et sa responsabilité dans son propre salut. Il aboutit ainsi à une sorte de dynamique pénitentielle, très inspirée de Grégoire, bien que fidèle au schéma de Cassien des vices et des vertus. En fin de compte, la morale pragmatique qu'il proposait était bien adaptée à la vie publique du groupe social des optimates, qui pouvaient eux aussi accéder aux portes du ciel, malgré les péchés inhérents à leur dignitas.

81. De virtutibus et vitiis, lettre introductive, MGH, Ep. IV, p. 464-465, trad. J. CHeLINI, L'aube du Moyen Âge..., p. 80.

82. Jonas d'Orléans, Vita sancti Hucberti, ASS Novembre, t. I, c. 817 C. 
Il est bien difficile d'évaluer l'impact de ce genre de traité sur la conduite des grands du royaume carolingien. Tout au plus peut-on signaler qu'Alcuin lui-même tenait le destinataire de son ouvrage dans la plus haute estime et le considérait comme « un homme parfait et un juge incorruptible ${ }^{83}$ ». Il prêchait donc quelqu'un qu'il savait disposé à l'écouter. Par ailleurs, mais sans chercher de lien de cause à effet avec le traité d'Alcuin, il faut signaler que les traités des vices et des vertus devinrent rapidement un instrument de la pastorale de l'Église carolingienne : les capitula ecclesiastica promulgués entre 810 et 813 et introduits plus tard dans la collection d'Anségise prévoyaient ainsi que chaque prêtre possédât une collection de capitula des vertus et vices, pour s'informer lui-même, mais aussi pour la prédication aux fidèles ${ }^{84}$. Ce passage dans la norme explique le développement extraordinaire du genre des traités des vices et vertus pendant tout le Moyen Âge.

83. Alc. Ep. 249, MGH Ep. Kar. aevi II, p. 402, 5 : Illorum siquidem conversationem et vitam a viro perfecto et judice incorrupto et misso fideli Widone audire potestis.

84. Capitula ecclesiastica 810-813, MGH Cap I, p. 179 : Ut unusquisque presbyter capitula habeat de maioribus vel de minoribus vitiis, per quae cognoscere valeat vel praedicare subditis suis, ut caveant ab insidiis diaboli, cf. R. McKITTERICK, The Frankish church..., p. 160. 


\section{RESUME}

À la fin de sa vie, Alcuin rédigea pour le comte Guy de Bretagne un Livre des vertus et des vices, qui n'est pas encore un miroir, car il ne propose pas de réflexion sur l'état laïc en lui-même. Il s'agit d'un liber manualis, qui veut montrer comment un grand, engagé dans la vie politique et militaire et ayant peu de temps pour les affaires religieuses, peut faire son salut, grâce à une morale efficace. Le modèle proposé à ce soldat du Christ est de type monastique : c'est celui du combat spirituel des vertus et des vices, parallèle au combat matériel qu'il mène au service de l'empire chrétien. Les vices qui le menacent étant semblables à ceux qui guettent le moine, Alcuin reprend la liste des vices proposée par Cassien, y compris l'acédie, devenue oisiveté poussant à l'inaction. Il propose une morale active, dans laquelle les vertus sont mises en pratique dans l'exercice de la fonction publique, pour faire régner l'ordre, la paix et la justice, en luttant contre les vices inhérents à la vie de cour. Le manuel fait ainsi passer dans le milieu aristocratique les notions de base de la culture ecclésiastique, en insérant le mode de vie laïc dans un modèle chrétien.

\section{ABSTRACT}

At the end of his life, Alcuin wrote on behalf of count Guy de Bretagne a Book of virtues and vices, that is not really a lay mirror, because it doesn't propose a global reflection on laity. It is in fact a liber manualis, that tries to show how a frankish magnate, committed in the political and military life and having little time for religious matters, can ensure his salvation, thanks to an efficient ethics. The model proposed to the Christ's soldier is a monastic one : it is the spiritual fight between virtues and vices, parallel to the material fight that he leads to the service of the Christian empire. The vices that threaten him being similar to those that befall the monk, Alcuin takes Cassian's list of vices, including acedia, turned into idleness, leading to inactivity. He proposes an active ethics, in which the virtues are put in practice in the exercise of the public service, to make order, peace and justice reign, while fighting against the inherent vices of the life at court. The handbook introduces thus in the aristocratic environment basic notions of the ecclesiastical culture, while inserting the layman's way of life in a Christian model. 